\title{
Positive metacognitions about alcohol mediate the relationship between FKBP5 variability and problematic drinking in a sample of young women
}

This article was published in the following Dove Press journal:

Neuropsychiatric Disease and Treatment

\author{
Wojciech Łukasz Dragan' \\ Wojciech Domozych' \\ Piotr M Czerski² \\ Małgorzata Dragan ${ }^{3}$ \\ 'The Interdisciplinary Centre for \\ Behavioural Genetics Research, \\ Faculty of Psychology, University of \\ Warsaw, Warsaw, Poland; ${ }^{2}$ Laboratory \\ of Psychiatric Genetics, Department \\ of Psychiatry, Poznan University of \\ Medical Sciences, Poznan, Poland; \\ ${ }^{3}$ Faculty of Psychology, University of \\ Warsaw, Warsaw, Poland
}

Background: Previous research has shown that polymorphisms in the FKBP5 gene are related to some psychiatric conditions, including alcohol dependence. These relationships are moderated by the level of adverse childhood experiences that one has undergone. Maladaptive metacognition, associated with symptoms of psychiatric disorders and disturbed emotional self-regulation, is also a strong predictor of problematic alcohol use. Recent studies suggest that maladaptive metacognitions may be part of the developmental pathway from childhood abuse to drinking problems. This study attempted to identify relationships between FKBP5 polymorphisms and metacognitions about the positive effects of alcohol use and problematic drinking in a group differing in levels of childhood trauma.

Methods: The sample studied was composed of 502 female participants aged 18-25 years $(\mathrm{M}=21.78 ; \mathrm{SD}=1.84)$. Positive metacognitions about alcohol use were measured with the Positive Alcohol Metacognitions Scale (PAMS) and problematic drinking was gauged using the WHO Alcohol Use Disorders Identification Test. Levels of childhood adverse experiences were determined with the use of the Childhood Questionnaire. A total of 18 single-nucleotide polymorphisms (SNPs) in the FKBP5 gene were genotyped.

Results: We did not find any interaction between the gene and childhood trauma on problematic drinking or metacognitions. However we identified a strong main effect of two SNPs of the FKBP5 gene - rs755658 and rs1334894 - on the PAMS subscale measuring positive metacognitive beliefs about emotional self-regulation. We also found nominally significant relations of several other SNPs with metacognitions and problematic drinking. Additionally, we showed that positive alcohol metacognitions mediate the relationship between problematic drinking and both rs755658 and rs1334894.

Conclusion: Our results may shed some light on the biological underpinnings of the developmental pathway leading to problematic drinking through maladaptive metacognitions.

Keywords: maladaptive metacognition, FKBP5, problematic drinking, adverse childhood experiences

\section{Introduction}

The hypothalamic-pituitary-adrenal (HPA) axis is a primary component of the stress response system. Glucocorticoids - major substances released by the HPA axis - as well as their receptors and binding proteins are critical for the regulation of the neuroendocrine system. ${ }^{1}$ The dysregulation of the HPA axis is implicated in the

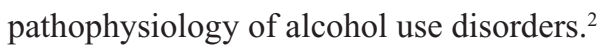

FK506-binding protein 5 (FKBP5) is a co-chaperone of heat-shock protein 90, which regulates glucocorticoid receptor (GR) sensitivity to cortisol and the efficiency of negative feedback in the HPA axis. ${ }^{3}$ Increased expression of the Fkbp5 gene has
Correspondence: Wojciech Łukasz Dragan

The Interdisciplinary Centre for Behavioural Genetics Research, Faculty of Psychology, University of Warsaw, Stawki Str 5/7, 00-183 Warsaw, Poland Email wdragan@psych.uw.edu.pl (c) (1) (5) 2018 Dragan et al. This work is published and licensed by Dove Medical Press Limited. The full terms of this license are available at https://www.dovepress.com/terms.php cc) ${ }_{\mathrm{BY}} \mathrm{NC}$ and incorporate the Creative Commons Atribution - Non Commercial (unported, v3.0) License (http:///creativecommons.org/licenses/by-nc/3.0/). By accessing the work you hereby accept the Terms. Non-commercial uses of the work are permitted without any further permission from Dove Medical Press Limited, provided the work is properly attributed. For permission for commercial use of this work, please see paragraphs 4.2 and 5 of our Terms (https://www.dovepress.com/terms.php). 
been observed in the hippocampus following chronic social defeat in mice. ${ }^{4}$ Moreover, changes in Fkbp 5 expression can be induced by administering alcohol. ${ }^{5}$

FKBP5 variation has been shown to interact with exposure to childhood abuse to increase the risk of posttraumatic stress disorder (PTSD) and major depression. A recent meta-analysis ${ }^{6}$ showed that individuals who carry the T allele of rs1360780, the $\mathrm{C}$ allele of rs3800373, or the T allele of rs9470080 who had been exposed to environmental risk had higher risks for both disorders. Alcohol dependence has been reported to be comorbid with $\mathrm{PTSD}^{7}$ and major depression. ${ }^{8}$ Interestingly, Xie et $\mathrm{al}^{9}$ found that polymorphisms in FKBP5 are associated with greater comorbidity of alcoholism with PTSD. Moreover, FKBP5 variability was related to heroin dependence ${ }^{10}$ and severity of alcohol withdrawal. ${ }^{11}$ Recently, Qiu et al ${ }^{12}$ reported that two FKBP5 polymorphisms (rs1360780 and rs3800373) are related to lifetime alcohol use disorder symptoms and quantity of alcohol drinking among college students.

Wells's metacognitive model ${ }^{13}$ is a model of psychopathology which describes the mechanisms of the development of disorders in terms of non-adaptive and disturbed selfregulation. Based on its general assumptions, Spada et al ${ }^{14}$ proposed a metacognitive model of cognitive-emotional regulation in addictive behaviors. This model assumes, inter alia, that specific maladaptive beliefs about alcohol use and negative affect lead to alcohol use as a means of affect regulation. These beliefs are positive metacognitions conceptualized as a specific form of expectancy relating to the use of alcohol as a means of controlling and regulating cognition and emotion, for example, "drinking will help me reduce my worrying" or "drinking will improve my mood". Spada et $\mathrm{al}^{15}$ emphasize that although both metacognitive beliefs about alcohol use and alcohol expectancies can describe motivations for alcohol use, a crucial difference is that positive alcohol expectancies do not explicitly distinguish between cognitive and metacognitive belief domains. However, according to metacognitive theory, the key markers of psychopathology are beliefs pertaining to the metacognitive domain. Spada et $\mathrm{al}^{14}$ assume that positive metacognitions about alcohol use are particularly crucial in the initial stage of development of alcohol-related problems, while in further stages other processes increase in significance (such as negative metacognitive beliefs about the uncontrollability of emotions and thoughts). Furthermore, Spada and Wells ${ }^{16}$ found that positive metacognitive beliefs about both cognitive and emotional self-regulation are related to problem drinking behavior, as measured by the Alcohol Use Disorders Identification Test (AUDIT).
The concept of positive alcohol expectancies is similar to positive metacognitions about alcohol. Both concern emotional self-regulation. Additionally, positive metacognitions refer to the impact of alcohol on cognitive processes (problem-solving, thought control, attention regulation, and self-image control). ${ }^{17}$ Importantly, a recent twin study by Young-Wolff et $\mathrm{al}^{18}$ revealed that genetic influences explain positive alcohol expectancies to a greater extent the longer one has been drinking. Moreover, Handley et al ${ }^{19}$ reported that children with a specific haplotype of FKBP5 who experienced maltreatment are more prone to develop alcohol use disorders. The developmental pathway from childhood abuse to drinking problems includes symptoms of internalizing behaviors and alcohol expectancies. Taken together, these findings suggest that FKBP5 variation is implicated in the modulation of self-regulation processes related to proneness to alcohol use disorders. From previous studies, we know that some variants of FKBP5 confer the risk of several forms of psychopathology, including alcohol use disorders. We also know that variability in this gene can influence psychological factors related to risk of psychopathology - that is, personality traits ${ }^{20}$ and alcohol expectancies. Positive alcohol metacognitions representing the broad spectrum of beliefs about the impact of alcohol on cognitive and emotional self-regulation may alter drinking behaviors. ${ }^{17}$ Therefore we anticipate that polymorphisms in FKBP5 (especially those which have previously been associated with PTSD and major depression - that is, rs1360780, rs3800373, and rs9470080) will be related to positive alcohol metacognitions and consequently to problem drinking. Previous studies have clearly shown that individuals carrying some of the FKBP5 alleles are vulnerable to adverse environments. ${ }^{6}$ Therefore we also expect that the relationship between FKBP5 and positive alcohol metacognitions and problem drinking will be moderated by exposure to childhood trauma - that is, the relation between gene variation and alcohol-use-related variables would be present in the subsample with high levels of childhood trauma. To address these issues, we performed a series of association analyses using data gathered in a non-clinical sample of young women. We have focused on this group for several reasons. First, there are significant gender differences in alcohol-drinking patterns, and some risk factors seem to be more significant for women than men. ${ }^{21}$ Moreover, alcoholdrinking patterns crystallize at a young age and early adulthood is a period of life with a heightened risk of drinking among women, whose drinking tends to decline sharply later in life. Early adulthood is also associated with heightened 
risk of general psychopathology. Moreover, in the case of women, adverse childhood experiences are among the main risk factors for problem drinking (see, eg, Brady et $\mathrm{al}^{22}$ ). Therefore, it can be assumed that drinking to self-regulate might be an important, or even primary, mechanism related to problem drinking in this demographic.

\section{Materials and methods Participants}

A non-clinical sample of 502 young women was recruited via advertisements distributed mainly at academic sites and, additionally, through a social networking service (the Facebook pages of academic departments). Being a student of psychology was an exclusion criterion; inclusion criteria were gender (female) and age (18-25 years). A total of 54.6\% of participants reported having at least secondary education and the vast majority (96\%) were single. The mean age was $\mathrm{M}=21.78$ years $(\mathrm{SD}=1.84)$. All participants were Caucasian (99\% were Polish nationals). Participation in the study was voluntary and participants gave their informed consent in written form to the researchers. They completed the questionnaires individually and, after completing them, the participants were paid the equivalent of 10 USD. The study was approved by the Research Ethics Committee of the Faculty of Psychology, University of Warsaw.

\section{Measures}

Positive metacognitions about alcohol use were measured using the Positive Alcohol Metacognitions Scale (PAMS). The PAMS is a measure developed to assess positive metacognitions about alcohol use - a specific form of alcohol expectancy relating to the beliefs individuals hold about the effects of alcohol on cognition and emotion. ${ }^{16}$ It consists of two subscales: PAMS1, which concerns positive metacognitions about emotional self-regulation through drinking (eight items - eg, "Drinking reduces my anxious feelings" or "Drinking makes me more sociable"); and PAMS2, which concerns positive metacognitions about cognitive self-regulation through drinking (four items - eg, "Drinking makes my negative thoughts more bearable" or "Drinking makes me think more clearly"). The measure is scored using a 4-point likelihood scale which ranges from "do not agree" to "agree very much." Participants are asked to indicate to what extent they agree with the statements given. Results from validation studies ${ }^{23}$ suggest that the PAMS possesses good psychometric properties. In the current study, the total score and both subscales were used as indexes of positive metacognitions about alcohol use. Cronbach's alpha for the total PAMS score was 0.85 , for PAMS1 it was 0.85 , and for PAMS2 it was 0.64 .

In order to determine the intensity of problem drinking related to positive metacognitions about alcohol use, the widely known WHO AUDIT ${ }^{24}$ was used. The most common criterion used in research for determining problem drinking with this tool is a score of 8 or more. In the current study, Cronbach's alpha for the total AUDIT score was 0.81 .

Childhood abuse was measured using the Childhood Questionnaire (CQ). ${ }^{25}$ The CQ retrospectively measures the occurrence of 14 types of adverse experiences before the age of 14 years (eg, sexual abuse, physical abuse, poverty, the death of a parent). Some experiences were assessed separately for each parent, so the scale has a total of 18 possible experiences. For this study, we used the total CQ score, which is the sum of all adverse experiences indicated by the individual. The Cronbach's alpha for the total CQ score for this sample was 0.71 . The median of the total CQ score was used to distinguish participants with low vs high levels of adverse childhood experiences.

\section{Genotyping}

DNA was isolated from all participants. The biological material was obtained from saliva using the Oragene ${ }^{\circledR}$ saliva collection system (DNA Genotek Inc, Ottawa, ON, Canada) and extracted using the provided protocol. On the basis of existing literature and the HapMap database, 18 single-nucleotide polymorphisms (SNPs) in the FKBP5 gene were selected for individual genotyping: rs3800374, rs3800373, rs7757037, rs755658, rs9296158, rs9380524, rs7748266, rs1360780, rs4713902, rs1334894, rs9394309, rs4713904, rs9470079, rs9470080, rs4713916, rs3800372, rs10456432, and rs7751598. A MALDI-TOF mass spectrometry-based SNP genotyping technique was used (Sequenom MassARRAY iPLEX platform, Sequenom, San Diego, CA, USA). Only FKBP5 SNPs with minor allele frequency $(\mathrm{MAF}) \geq 5 \%$ and completeness $\geq 75 \%$ across the pooled data were included in further statistical analyses.

\section{Statistical analyses}

In order to examine the relationship between the selected SNPs and alcohol-use-related variables, we performed a series of association analyses based on a generalized linear model. First, we tested simple genetic effects using intensity of problem drinking (measured by AUDIT) and positive metacognitions about alcohol use (measured by PAMS) as dependent variables and age as a covariate. Second, we repeated all analyses using an interaction term (childhood abuse level: low vs high). All calculations were performed 
using the SNPassoc package ${ }^{26}$ for R. Analyses in which simple genetic effects were tested were made using five genetic models: codominant, dominant, recessive, overdominant, and log-additive. Analyses with an interaction term were done for four genetic models: codominant, dominant, recessive, and overdominant. The Akaike Information Criterion (AIC) produced by SNPassoc was used to identify the best-fitting model. The corrected level of significance was set at 0.00244 and was drawn from Li and Ji's $\mathrm{V}_{\text {eff, }}{ }^{27}$ based on a Spearman correlation matrix produced by pairwise exclusion of missing observations. SNPassoc was also used to estimate the Hardy-Weinberg equilibrium (HWE) $P$-value for all selected SNPs.

When a significant association between a SNP and both problem drinking and positive alcohol metacognition was detected, a mediation model was tested. Mediation analyses were performed using IBM SPSS software and the PROCESS macro for IBM SPSS. ${ }^{28}$ Power analysis was performed with the use of Quanto software.

\section{Results}

The mean result for PAMS was $\mathrm{M}=25.72(\mathrm{SD}=6.62$, $\mathrm{min}=12$, $\max =45)$. The mean result on the AUDIT was $M=7.04$ $(\mathrm{SD}=4.63, \min =0, \max =24)$. The results of 158 participants (31.5\%) exceeded the AUDIT cutoff of 8 points. Pearson's correlation coefficient between PAMS and AUDIT scores was $r=0.47$, significant at $P<0.001$. The mean result for CQ was $\mathrm{M}=2.18(\mathrm{SD}=2.17, \min =0, \max =11)$, the median was 2. Table 1 shows the descriptive statistics of AUDIT and PAMS in subgroups distinguished using the median value of the CQ.

Genotype distributions for most of the polymorphisms were in line with the HWE. Exceptions were rs7757037 and rs3800372 (see Supplementary materials). The power

Table I Descriptive statistics of AUDIT and PAMS in subgroups differing in levels of $C A$

\begin{tabular}{l|l|l|l}
\hline & $\begin{array}{l}\text { Low level of CA } \\
\mathbf{N}=\mathbf{2 3 4}\end{array}$ & $\begin{array}{l}\text { High level of CA } \\
\mathbf{N}=\mathbf{2 6 8}\end{array}$ & t-test results \\
\hline AUDIT & $\mathrm{M}=6.1 \mathrm{I}$ & $\mathrm{M}=7.85$ & $\mathrm{SD}=4.94$ \\
& $\mathrm{SD}=4.06$ & $\mathrm{M}=26.82$ & $\begin{array}{l}\mathrm{t}(498.2 \mathrm{I})=-4.32 \\
\mathrm{P}<0.00 \mathrm{I}\end{array}$ \\
\hline PAMS & $\mathrm{M}=24.46$ & $\mathrm{SD}=6.59$ & $\mathrm{t}(498)=-4.02$ \\
& $\mathrm{SD}=6.44$ & $\mathrm{M}=20.6 \mathrm{I}$ & $\mathrm{P}<0.00 \mathrm{I}$ \\
\hline PAMSI & $\mathrm{M}=18.93$ & $\mathrm{SD}=5.46$ & $\mathrm{t}(498)=-3.47$ \\
& $\mathrm{SD}=5.36$ & $\mathrm{M}=6.19$ & $\mathrm{P}<0.0 \mathrm{I}$ \\
\hline PAMS2 & $\mathrm{M}=5.55$ & $\mathrm{SD}=\mathrm{I.92}$ & $\mathrm{t}(500)=-3.96$ \\
& $\mathrm{SD}=\mathrm{I} .72$ & $\mathrm{P}<0.00 \mathrm{I}$ \\
\hline
\end{tabular}

Abbreviations: AUDIT, Alcohol Use Disorders Identification Test; CA, childhood abuse; PAMS, Positive Alcohol Metacognitions Scale. analysis indicated that our study had $62 \%$ power to detect genetic effects and $32 \%$ power to detect interaction effects (alpha=0.05).

Simple genetic effects were tested in the first association analysis step. We identified a significant association between SNP rs755658 and the total PAMS score. The two best-fitted models were the dominant $(P=0.00188, \mathrm{AIC}=3226)$ and logadditive $(P=0.0014, \mathrm{AIC}=3225)$ models. This association was mainly due to the relation of the SNP with the PAMS1 score (dominant model: $P=0.001812, \mathrm{AIC}=3040$; log-additive model: $P=0.00126, \mathrm{AIC}=3040)$. We also identified a significant association between SNP rs1334894 and the total PAMS score. The two best-fitted models were the dominant $(P=0.00166, \mathrm{AIC}=3226)$ and log-additive $(P=0.00131$, $\mathrm{AIC}=3225$ ) models. Again, this association was due to the relation of the SNP with the PAMS1 score (dominant model: $P=0.00167, \mathrm{AIC}=3040 ; \log$-additive model: $P=0.00117$, $\mathrm{AIC}=3039$ ). We did not identify any significant relations between PAMS2 score and either rs755658 or rs1334894. The medians and standard errors of total PAMS and PAMS1 scores for groups distinguished on the basis of rs755658 and rs1334894 are presented in Table 2 and Figure 1. We also identified a significant relation between SNP rs4713916 and PAMS2 score. The best-fitted model was log-additive $(P=0.00225, \mathrm{AIC}=1984)$. The medians and standard errors of PAMS2 scores for groups distinguished on the basis of rs4713916 are presented in Table 2 and Figure 1. We also found nominally significant (not significant after multiple testing correction) associations of:

- rs3800373 with PAMS2 score (dominant model: $P=0.024$, $\mathrm{AIC}=1999)$;

- rs7751598 with PAMS2 score (dominant model: $P=0.014$, $\mathrm{AIC}=1998$ );

- rs1360780 with PAMS2 score (dominant model: $P=0.014$, $\mathrm{AIC}=1998)$;

Table 2 Descriptive statistics of PAMS total score and PAMSI in subgroups differing in genotype for rs755658 and rs 1334894

\begin{tabular}{|c|c|c|c|c|c|c|c|}
\hline \multirow[t]{2}{*}{ SNP } & \multirow[t]{2}{*}{ Genotype } & \multicolumn{3}{|c|}{ PAMS total score } & \multicolumn{3}{|c|}{ PAMSI } \\
\hline & & $\mathbf{n}$ & $\mathbf{M}$ & SE & $\mathbf{n}$ & $\mathbf{M}$ & SE \\
\hline \multirow[t]{4}{*}{ rs755658 } & $\mathrm{g} / \mathrm{g}$ & 405 & 25.31 & 0.32 & 405 & 19.49 & 0.266 \\
\hline & $\mathrm{a} / \mathrm{g}$ & 74 & 27.47 & 0.86 & 74 & 21.26 & 0.694 \\
\hline & $a / a$ & 9 & 29.44 & 1.676 & 9 & 23.11 & 1.504 \\
\hline & $a / g-a / a$ & 83 & 27.69 & 0.788 & 83 & 21.46 & 0.64 \\
\hline \multirow[t]{4}{*}{ rsI334894 } & $\mathrm{c} / \mathrm{c}$ & 404 & 25.30 & 0.321 & 404 & 19.49 & 0.267 \\
\hline & $c / t$ & 75 & 27.49 & 0.846 & 75 & 21.27 & 0.68 \\
\hline & $\mathrm{t} / \mathrm{t}$ & 9 & 29.44 & 1.676 & 9 & 23.11 & 1.504 \\
\hline & $c / t-t / t$ & 84 & 27.70 & 0.774 & 84 & 21.46 & 0.629 \\
\hline
\end{tabular}


A

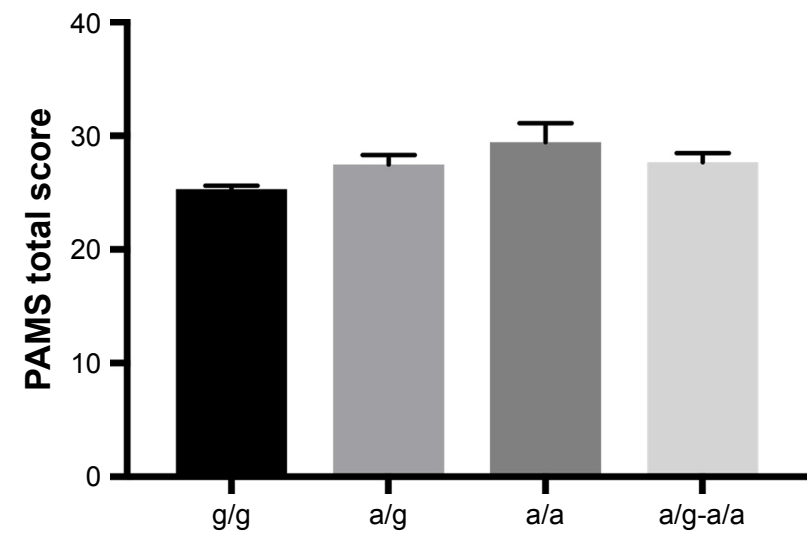

C

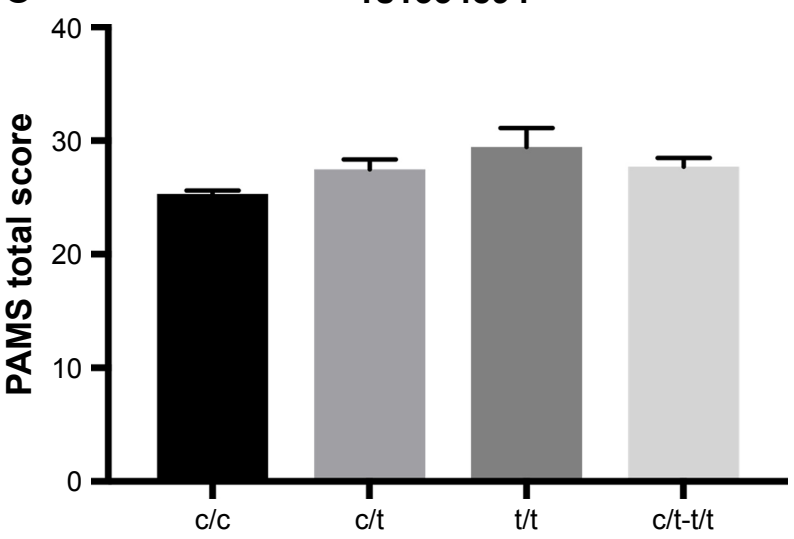

B

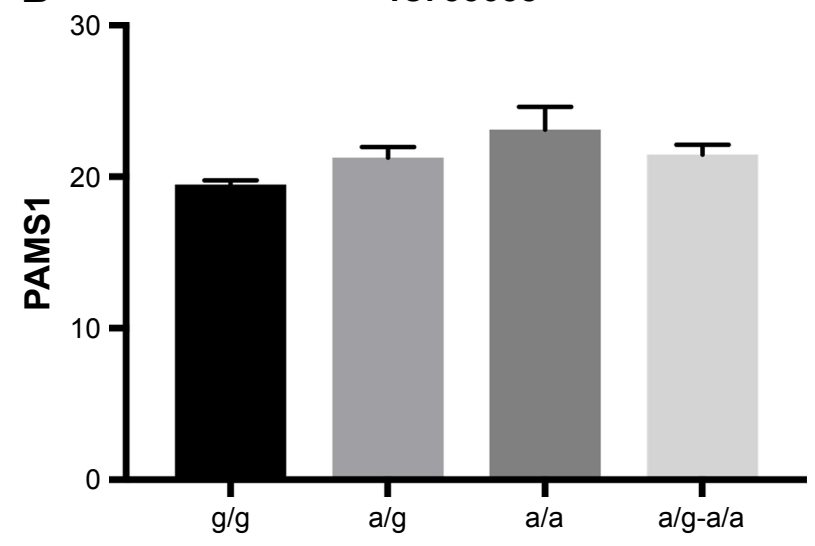

D

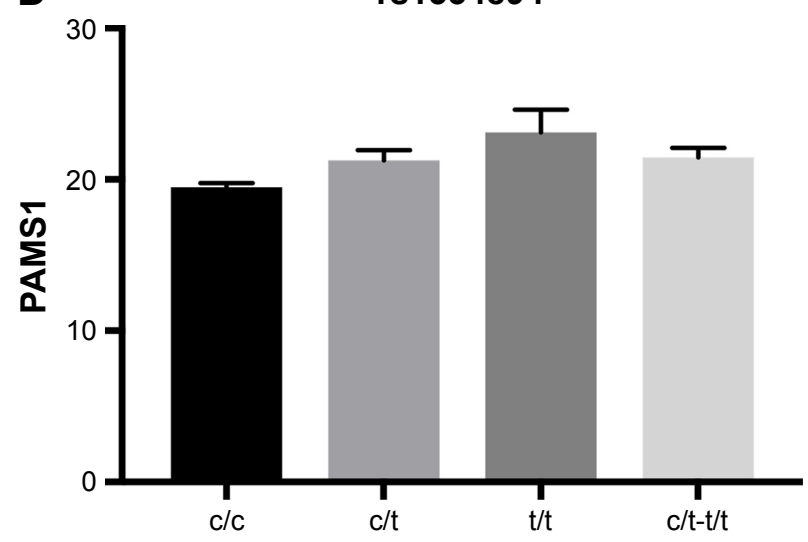

Figure I Means and standard errors of PAMS total and PAMSI scores in groups differing in rs755658 and rs I 334894 .

Notes: (A) Descriptive statistics for the association of rs755658 and PAMS total score; (B) descriptive statistics for the association of rs755658 and PAMSI score; (C) descriptive statistics for the association of rs I334894 and PAMS total score; (D) descriptive statistics for the association of rs I334894 and PAMSI score.

- rs9394309 with total PAMS score (recessive model: $P=0.01, \mathrm{AIC}=3223$ ), PAMS1 score (recessive model: $P=0.023$, AIC $=3039)$, and with PAMS2 score $(\log$ additive model: $P=0.0033, \mathrm{AIC}=1992$ );

- rs4713904 with total PAMS score (log-additive model: $P=0.017$, AIC $=3230$ ) and with PAMS2 score (logadditive model: $P=0.0042, \mathrm{AIC}=1995)$;

- rs9470080 with total PAMS score (recessive model: $P=0.04, \mathrm{AIC}=3231)$ and with PAMS2 score (log-additive model: $P=0.0063$, AIC $=1996)$; as well as

- rs4713916 with total PAMS score (log-additive model: $P=0.0085, \mathrm{AIC}=3221$ ) and with PAMS1 score (recessive model: $P=0.022$, AIC=3039).

Detailed results for all SNPs are presented in the Supplementary materials.

We also identified nominally significant associations of rs755658 and rs1334894 with problematic drinking measured by AUDIT. In both the cases, dominant and log-additive models fit best (respectively, for rs755658: $P=0.048, \mathrm{AIC}=2887$ and $P=0.024, \mathrm{AIC}=2885$; and for rs 1334894: $P=0.032$, $\mathrm{AIC}=2886$ and $P=0.016, \mathrm{AIC}=2885$ ).

In the second step, we tested interaction effects. We did not identify significant relations between any of the tested SNPs and metacognitive beliefs or alcohol use. However, the analysis revealed nominally significant associations of AUDIT score with:

- rs7757037 (dominant model, $P=0.02$ );

- rs4713902 (recessive model, $P=0.047$ ); and

- rs10456432 (dominant model, $P=0.004$ ).

Detailed results for all SNPs are presented in the Supplementary materials.

Finally, we performed mediation analysis. We tested a model in which metacognitive beliefs mediated the relation between SNPs (rs755658 and rs1334894) and problematic drinking. Although, the log-additive model of inheritance was best fitted to the data, we grouped genotypes according to the dominant model as this was more useful in the mediation analyses. Mediation analyses with 5,000 bootstrapped 
Table 3 Analyses of mediation of the relationship between genotype and AUDIT total score

(a) rs755658

\begin{tabular}{|c|c|c|c|c|c|c|c|c|c|c|}
\hline \multirow[t]{2}{*}{ Mediator } & \multicolumn{5}{|c|}{ PAMS total score } & \multicolumn{5}{|c|}{ PAMSI } \\
\hline & Effect & SE & $95 \% \mathrm{Cl}$ & $\mathbf{t}$ & $P$-value & Effect & SE & $95 \% \mathrm{CI}$ & $\mathbf{t}$ & $P$-value \\
\hline Total effect & 1.08 & 0.554 & $(-0.004 ; 2.17)$ & 1.96 & $0.05 I$ & 1.08 & 0.554 & $(-0.004 ; 2.17)$ & 1.96 & 0.051 \\
\hline \multirow[t]{2}{*}{ Direct effect } & 0.293 & 0.499 & $(-0.69 ; 1.27)$ & 0.59 & 0.556 & 0.33 & 0.505 & $(-0.66 ; 1.03)$ & 0.66 & 0.509 \\
\hline & Effect & SE & $\begin{array}{l}\text { Bootstrapped } \\
95 \% \mathrm{Cl}\end{array}$ & \multicolumn{2}{|c|}{ Significance } & Effect & SE & $\begin{array}{l}\text { Bootstrapped } \\
95 \% \mathrm{Cl}\end{array}$ & \multicolumn{2}{|c|}{ Significance } \\
\hline Indirect effect & 0.79 & 0.289 & $(0.272 ; 1.394)$ & \multicolumn{2}{|c|}{ Significant } & 0.75 & 0.27 & $(0.24 ; 1.32)$ & \multicolumn{2}{|c|}{ Significant } \\
\hline
\end{tabular}

(b) rs 1334894

\begin{tabular}{|c|c|c|c|c|c|c|c|c|c|c|}
\hline \multirow[t]{2}{*}{ Mediator } & \multicolumn{5}{|c|}{ PAMS total score } & \multicolumn{5}{|c|}{ PAMSI } \\
\hline & Effect & SE & $95 \% \mathrm{Cl}$ & $\mathbf{t}$ & $P$-value & Effect & SE & $95 \% \mathrm{Cl}$ & $\mathbf{t}$ & $P$-value \\
\hline Total effect & 1.17 & 0.55 & $(0.088 ; 2.25)$ & 2.12 & 0.034 & 1.17 & 0.55 & $(0.088 ; 2.25)$ & 2.12 & 0.034 \\
\hline \multirow[t]{2}{*}{ Direct effect } & 0.376 & 0.496 & $(-0.6 ; 1.35)$ & 0.75 & 0.449 & 0.42 & 0.503 & $(-0.57 ; 1.4 \mathrm{I})$ & 0.83 & 0.405 \\
\hline & Effect & SE & $\begin{array}{l}\text { Bootstrapped } \\
95 \% \mathrm{Cl}\end{array}$ & \multicolumn{2}{|c|}{ Significance } & Effect & SE & $\begin{array}{l}\text { Bootstrapped } \\
95 \% \mathrm{Cl}\end{array}$ & \multicolumn{2}{|c|}{ Significance } \\
\hline Indirect effect & 0.79 & 0.28 & $(0.3 ; 1.38)$ & \multicolumn{2}{|c|}{ Significant } & 0.75 & 0.26 & $(0.26 ; 1.29)$ & \multicolumn{2}{|c|}{ Significant } \\
\hline
\end{tabular}

Abbreviations: AUDIT, Alcohol Use Disorders Identification Test; PAMS, Positive Alcohol Metacognitions Scale.

resamples were performed using model 4 of PROCESS for IBM SPSS $^{28}$ with age as a covariate. For rs 755658 as predictor and PAMS total score as a mediator, we observed neither total nor direct effects of SNP on the outcome. However, we observed a significant indirect effect, which suggests mediation. The final model explained almost $22 \%$ of variance $-R^{2}=0.217, F(3,484)=44.88, P<0.001$.

For rs 755658 as a predictor and PAMS1 score as a mediator, we also did not observe a total or direct effect of SNP on problematic drinking. However we observed an indirect effect. The final model explained almost $20 \%$ of variance $R^{2}=0.196, F(3,484)=39.32, P<0.001$.

For rs1334894 as a predictor and PAMS total score as a mediator, we observed total and indirect effects of SNP on the outcome variable. We did not observe a direct effect, which indicates full mediation. The final model explained almost $22 \%$ of variance $-R^{2}=0.218, F(3,484)=44.97, P<0.001$. The same effect pattern was observed for rs 1334894 as a predictor and PAMS1 score as a mediator. The final model explained almost $20 \%$ of variance $-R^{2}=0.196, F(3,484)=39.43$, $P<0.001$. Results of the mediation analyses are shown in Table 3.

\section{Discussion}

The metacognitive model of psychopathology, ${ }^{13}$ which has also been applied to addictive behaviors, ${ }^{14}$ underlines the role of meta-cognitive mechanisms in disturbed self-regulation. Identifying the genetic bases of self-regulation disorders seems to be critical for understanding the mechanisms underlying their development. The present study aimed at identifying relations of polymorphisms in the FKBP5 gene with positive alcohol metacognitions and problematic drinking. We also expected that the level of childhood trauma would moderate these associations. We identified strong associations between positive metacognitive beliefs about emotional self-regulation and two SNPs - rs755658 and rs1334894 - and between positive metacognitive beliefs about cognitive self-regulation and rs4713916. We also identified nominally significant (not significant after multiple comparison adjustment) relations of positive alcohol metacognition with rs3800373, rs7751598, rs1360780, rs9394309, rs4713904, rs9470080, and rs4713916. Moreover, problematic drinking was related to rs755658 and rs1334894 at a nominally significant level. We tested mediation models and found that positive metacognitive beliefs about emotional self-regulation mediate the relationship of rs 755658 and rs1334894 with problematic drinking. As mentioned previously, a meta-analysis ${ }^{6}$ found that three polymorphisms in FKBP5 (rs1360780, rs3800373, and rs9470080) are related to major depression and PTSD. Moreover, Handley et $\mathrm{al}^{19}$ recently showed that the specific developmental pathway leading to alcohol dependence through positive expectancies toward alcohol use is influenced by $F K B P 5$ variability. Our findings are in line with the results of Handley et al, ${ }^{19}$ showing that FKBP5 may have an impact on the development of problematic drinking through emotional self-regulatory processes. As mentioned above, positive expectancies toward alcohol and positive alcohol metacognitions shared the content of emotional self-regulation 
(we found that only the PAMS1 score, which covers this aspect of metacognition, mediated the relation between FKBP5 and problematic drinking). Interestingly, FKBP5 variability was related to maladaptive regulation of the HPA axis resulting in hypercortisolism. ${ }^{3}$ Alcoholic patients show blunted stress-induced cortisol responses and increased negative emotions. ${ }^{29}$ There are several possible pathways leading from heightened secretion of cortisol to emotional dysregulation and the development of beliefs about the positive effects of alcohol as a means of affect regulation. One of them includes amygdala hyperactivation which creates a negative response bias to emotional stimuli. ${ }^{30}$ Another one incorporates hippocampal shape alterations ${ }^{31}$ which may influence memory formation. ${ }^{32}$ Beresford et $\mathrm{al}^{33}$ showed that hypercortisolism in alcohol dependence is related to hippocampal volume loss. FKBP5 variability is also related to the temperamental trait of harm avoidance, ${ }^{20}$ which may be the basis of the development of positive expectancies toward alcohol use. ${ }^{34}$

As in previous studies, we expected that the level of childhood adverse experiences would moderate the relation between FKBP5 and positive alcohol metacognition and problematic drinking. Likely due to the sample size and insufficient power of the study, we were not able to detect significant interaction effects. However, we found nominally significant associations of three SNPs (rs7757037, rs4713902, and rs10456432) and problematic drinking among women who had experienced elevated levels of childhood adverse experiences.

Despite some obvious limitations of this study (eg, the relatively small and gender-homogeneous sample) which restrict the interpretations of our results, we believe that these results can contribute to the understanding of processes related to the development of maladaptive metacognition associated with problematic alcohol use. Although this problem is not yet sufficiently researched, it is assumed that early stress and social processes related to the development of self-regulation skills are among the most significant factors associated with alcohol abuse. ${ }^{35}$ Our study shows that genetic factors might also play an important role, increasing the risk of disturbances in self-regulation.

\section{Acknowledgments}

This study was supported by the Ministry of Science and Higher Education Iuventus Plus (grant no 0105/IP3/2011/71) and a National Science Center grant (research project no 2011/01/B/HS6/01912).

\section{Disclosure}

The authors report no conflicts of interest in this work.

\section{References}

1. Godoy LD, Rossignoli MT, Pereira PD. A comprehensive overview on stress neurobiology: basic concepts and clinical implications. Front Hum Neurosci. 2018;12:127.

2. Stephens MA, Wand G. Stress and the HPA axis: role of glucocorticoids in alcohol dependence. Alcohol Res. 2012;34(4):468-483.

3. Binder EB. The role of FKBP5, a co-chaperone of the glucocorticoid receptor in the pathogenesis and therapy of affective and anxiety disorders. Psychoneuroendocrinology. 2009;34(Suppl 1):S186-S195.

4. Wagner KV, Marinescu D, Hartmann J, et al. Differences in FKBP51 regulation following chronic social defeat stress correlate with individual stress sensitivity: influence of paroxetine treatment. Neuropsychopharmacology. 2012;37(13):2797-2808.

5. Kerns RT, Ravindranathan A, Hassan S, et al. Ethanol-responsive brain region expression networks: implications for behavioral responses to acute ethanol in DBA/2J versus C57BL/6J mice. J Neurosci. 2005; 25(9):2255-2266.

6. Wang Q, Shelton RC, Dwivedi Y. Interaction between early-life stress and FKBP5 gene variants in major depressive disorder and post-traumatic stress disorder: A systematic review and meta-analysis. J Affect Disord. 2018;225:422-428.

7. Blanco C, Xu Y, Brady K, Pérez-Fuentes G, Okuda M, Wang S. Comorbidity of posttraumatic stress disorder with alcohol dependence among US adults: results from National Epidemiological Survey on Alcohol and Related Conditions. Drug Alcohol Depend. 2013;132(3):630-638.

8. Brière FN, Rohde P, Seeley JR, Klein D, Lewinsohn PM. Comorbidity between major depression and alcohol use disorder from adolescence to adulthood. Compr Psychiatry. 2014;55(3):526-533.

9. Xie P, Kranzler HR, Poling J, et al. Interaction of FKBP5 with childhood adversity on risk for post-traumatic stress disorder. Neuropsychopharmacology. 2010;35(8):1684-1692.

10. Levran O, Peles E, Randesi M, et al. Stress-related genes and heroin addiction: a role for a functional FKBP5 haplotype. Psychoneuroendocrinology. 2014;45:67-76.

11. Huang MC, Schwandt ML, Chester JA, et al. FKBP5 moderates alcohol withdrawal severity: human genetic association and functional validation in knockout mice. Neuropsychopharmacology. 2014;39(8): 2029-2038.

12. Qiu B, Luczak SE, Wall TL, et al. The FKBP5 gene affects alcohol drinking in knockout mice and is implicated in alcohol drinking in humans. Int J Mol Sci. 2016;17(8):1271.

13. Wells A. Metacognitive Therapy for Anxiety and Depression. New York, USA: Guilford Press; 2009.

14. Spada MM, Caselli G, Nikčević AV, Wells A. Metacognition in addictive behaviors. Addict Behav. 2015;44:9-15.

15. Spada MM, Moneta GB, Wells A. The relative contribution of metacognitive beliefs and expectancies to drinking behaviour. Alcohol. 2007; 42(6):567-574.

16. Spada MM, Wells A. Metacognitive beliefs about alcohol use: Development and validation of two self-report scales. Addict Behav. 2008;33(4): 515-527.

17. Clark A, Tran C, Weiss A, Caselli G, Nikčević AV, Spada MM. Personality and alcohol metacognitions as predictors of weekly levels of alcohol use in binge drinking university students. Addict Behav. 2012; 37(4):537-540.

18. Young-Wolff KC, Wang P, Tuvblad C, Baker LA, Raine A, Prescott CA. Drinking experience uncovers genetic influences on alcohol expectancies across adolescence. Addiction. 2015;110(4):610-618.

19. Handley ED, Rogosch FA, Cicchetti D. From child maltreatment to emerging adult problem drinking: Identification of a multilevel internalizing pathway among African American youth. Dev Psychopathol. 2017;29(5):1807-1821.

20. Shibuya N, Suzuki A, Sadahiro R, et al. Association study between a functional polymorphism of FK506-binding protein 51 (FKBP5) gene and personality traits in healthy subjects. Neurosci Lett. 2010;485(3): 194-197. 
21. Nolen-Hoeksema S. Gender differences in risk factors and consequences for alcohol use and problems. Clin Psychol Rev. 2004;24(8):981-1010.

22. Brady KT, Back SE, Greenfield SF. Women and ddiction: A comprehensive handbook. New York: Guilford Press; 2009.

23. Spada MM, Wells A. Metacognitive beliefs about alcohol use: development and validation of two self-report scales. Addict Behav. 2008;33(4):515-527.

24. Babor TF, Hofmann M, Delboca FK, et al. Types of alcoholics, I. Evidence for an empirically derived typology based on indicators of vulnerability and severity. Arch Gen Psychiatry. 1992;49(8):599-608.

25. Hardt J, Egle UT, Engfer A. [The childhood questionnaire, a tool for describing the childhood relationship with the parents]. Der Kindheitsfragebogen, ein Instrument zur Beschreibung der erlebten Kindheitsbeziehung zu den Eltern. Zeitschrift für Differentielle und Diagnostische Psychologie. 2003;24(1):33-43. German.

26. González JR, Armengol L, Solé X, et al. SNPassoc: an R package to perform whole genome association studies. Bioinformatics. 2007;23(5): 654-655.

27. Li J, Ji L. Adjusting multiple testing in multilocus analyses using the eigenvalues of a correlation matrix. Heredity. 2005;95(3):221-227.

28. Hayes AF. Introduction to Mediation, Moderation, and Conditional Process Analysis A Regression-Based Approach. New York: Guilford Press; 2018
29. Sinha R, Fox HC, Hong KA, Bergquist K, Bhagwagar Z, Siedlarz KM. Enhanced negative emotion and alcohol craving, and altered physiological responses following stress and cue exposure in alcohol dependent individuals. Neuropsychopharmacology. 2009;34(5):1198-1208.

30. Holz NE, Buchmann AF, Boecker R, et al. Role of FKBP5 in emotion processing: results on amygdala activity, connectivity and volume. Brain Struct Funct. 2015;220(3):1355-1368.

31. Fani N, Gutman D, Tone EB, et al. FKBP5 and attention bias for threat: associations with hippocampal function and shape. JAMA Psychiatry. 2013;70(4):392-400.

32. Buss C, Wolf OT, Witt J, Hellhammer DH. Autobiographic memory impairment following acute cortisol administration. Psychoneuroendocrinology. 2004;29(8):1093-1096.

33. Beresford TP, Arciniegas DB, Alfers J, et al. Hypercortisolism in alcohol dependence and its relation to hippocampal volume loss. J Stud Alcohol. 2006;67(6):861-867.

34. Read JP, O'Connor RM. High- and low-dose expectancies as mediators of personality dimensions and alcohol involvement. J Stud Alcohol. 2006;67(2):204-214.

35. Myers SG, Wells A. Early trauma, negative affect, and anxious attachment: the role of metacognition. Anxiety Stress Coping. 2015;28(6): 634-649.
Neuropsychiatric Disease and Treatment

\section{Publish your work in this journal}

Neuropsychiatric Disease and Treatment is an international, peerreviewed journal of clinical therapeutics and pharmacology focusing on concise rapid reporting of clinical or pre-clinical studies on a range of neuropsychiatric and neurological disorders. This journal is indexed on PubMed Central, the 'PsycINFO' database and CAS,

\section{Dovepress}

and is the official journal of The International Neuropsychiatric Association (INA). The manuscript management system is completely online and includes a very quick and fair peer-review system, which is all easy to use. Visit http://www.dovepress.com/testimonials.php to read real quotes from published authors. 